\title{
Intramuscular Hydatid Cyst of Adductor Magnus: A Rare Isolated Presentation
}

\author{
Gupta V, Tayade A, Kale S
}

Department of Radiodiagnosis and Imaging, MGIMS, Sewagram, Maharashtra

\begin{abstract}
The muscular localization of the hydatid cyst is uncommon $(0.5$ to $5.4 \%)$ and it is usually secondary to hepatic or pulmonary disease. A case of intramuscular hydatid cyst in adductor magnus is reported, calling attention to this atypical localization of the disease, which should be taken into account in the practice of surgery. The absence of residual cysts in the most common sites confers a higher interest to the case reported in this work. Though hydatid disease is endemic in India, we present this case due to the rarity of site and typical ultrasonography and computed tomography picture.
\end{abstract}

Keywords: Hydatid Cyst, Intramuscular

\section{Introduction}

Although a primary hydatid cyst involving the musculoskeletal system is rare, it should be included in the differential diagnosis for any cystic soft tissue mass found in patients from areas where the disease is endemic. ${ }^{2}$ In humans, the most favored site for infestation is the liver $(65 \%)$ or the lungs $(25 \%)$; it rarely involves the brain, heart, bone, or other organs and primary muscular hydatid cysts comprise less than $0.5 \%$ of the cases in endemic populations. Primary Hydatid disease of the skeletal muscle without systemic involvement is rare. Bayram and Siricki reported the first intramuscular hydatid cysts in forearm region of 18 year old man. We report a case

Correspondence to: Dr. Vinit Gupta, Department of Radiodiagnosis and Imaging, MGIMS, Sewagram, Maharashtra Email:- dr.vinitgupta@gmail.com of a middle age male presenting with a swelling on the medial aspect of right thigh, the preoperative diagnosis of which was hydatid cyst on imaging findings.

\section{Case Report}

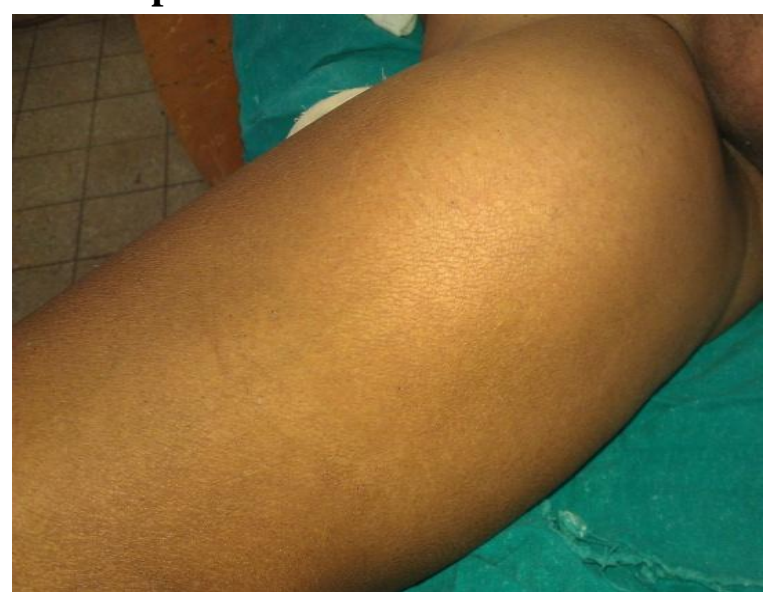

Fig. 1: Gross soft tissue swelling over medial aspect of thigh

A 48 year old male, non-vegeterian farmer dwelling in the rural area of central India, presented with a large swelling on the medial aspect of the right thigh since $17 \mathrm{yrs}$ which 


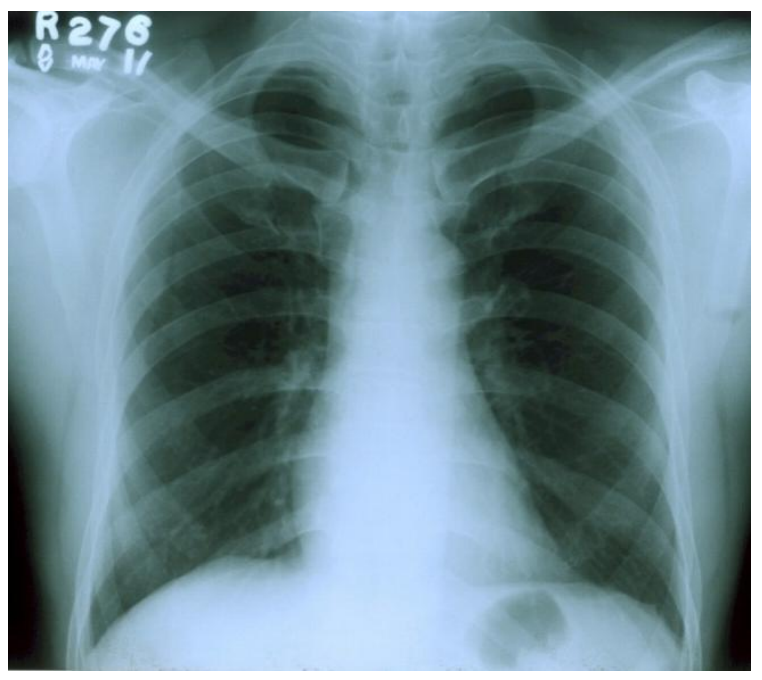

Fig. 2: Plain chest radiograph reveals no signs of hydatid dissemination.

was painless, insidious in onset, and gradually progressive with no history of weight loss. There were no pets in his house. On physical examination there was a single round to oval, non tender swelling of size 15 x $20 \mathrm{~cm}$, on the medial aspect of right thigh. (Fig.1) Swelling was fixed to the underlying muscle, having smooth surface, regular margins and was firm in consistency.

There were no other positive clinical findings. Routine laboratory tests were normal. Plain chest X-rays and abdominal Ultrasonography did not reveal any organ involvement. (Fig. 2)

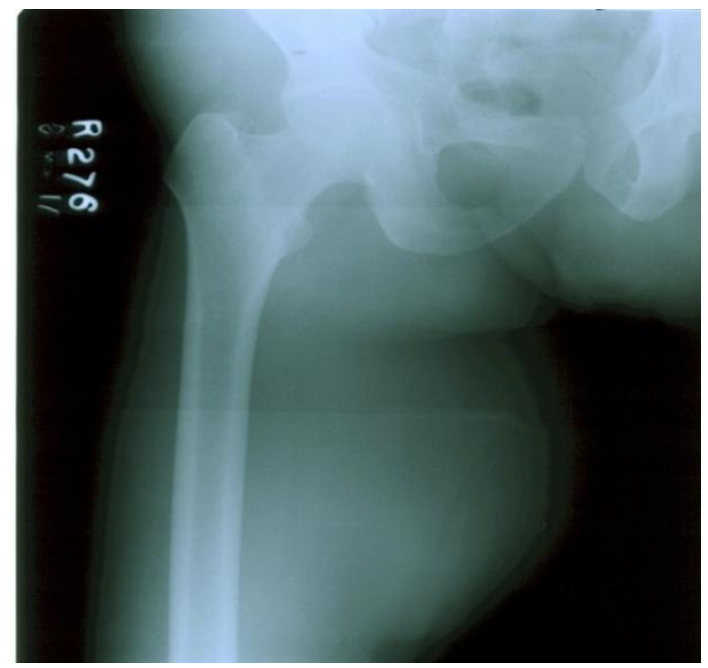

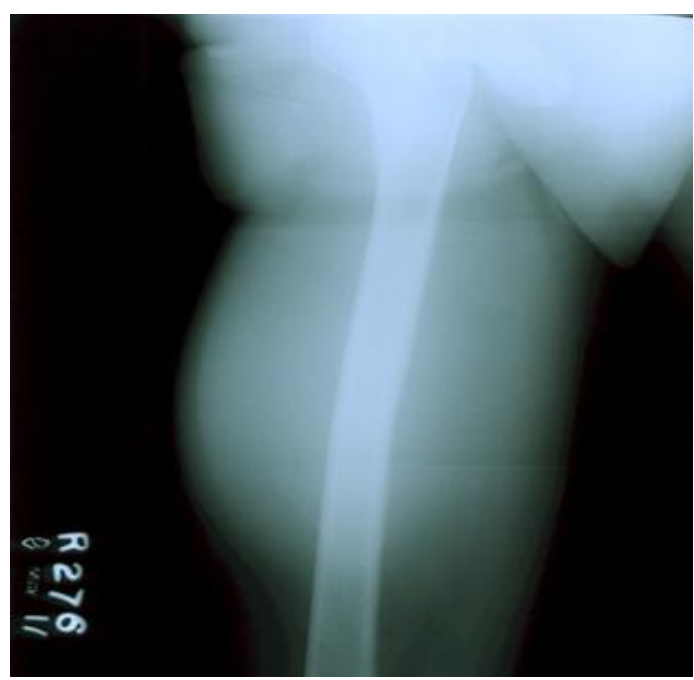

Fig. 3: Plain radiographs of thigh (Anteroposterior and lateral views) demonstrate soft tissue swelling on the medial aspect of thigh. No evidence of bony destruction or pressure effect seen suggestive of Soft tissue mass lesion.
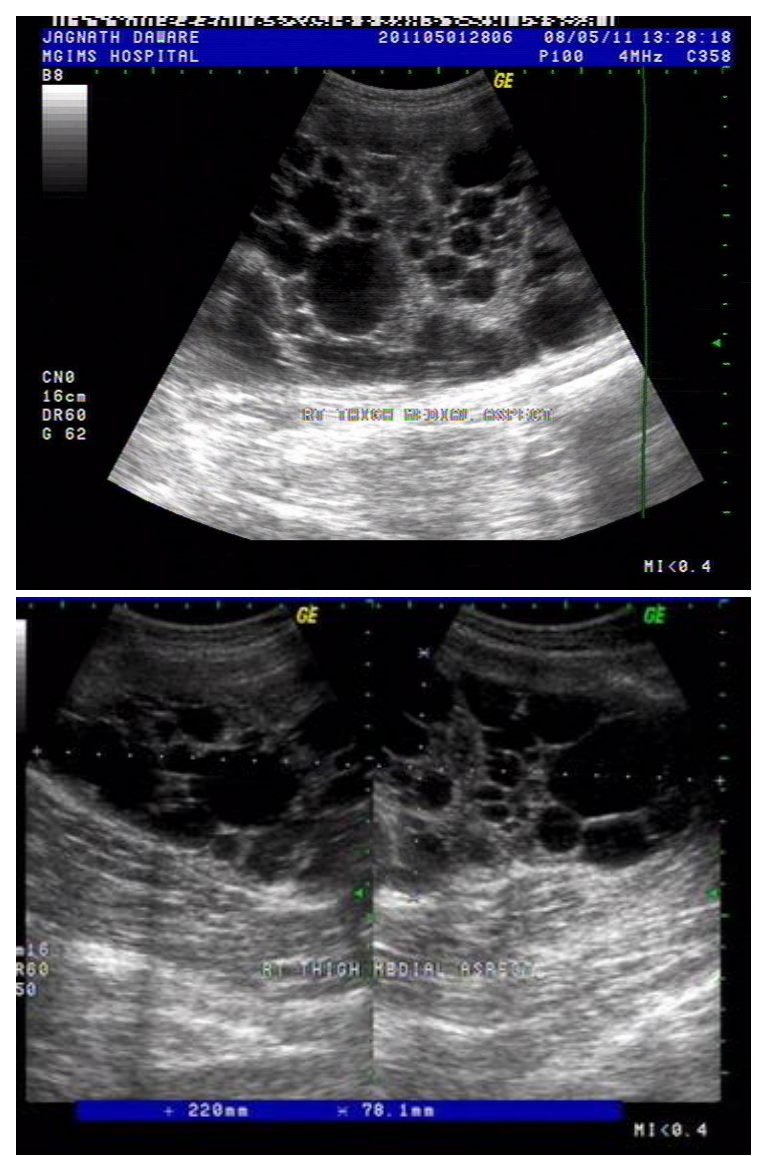

Fig. 4 a \& b: Gray scale images demonstrate multiple well defined round to oval cystic spaces in the muscle plane suggestive of daughter cysts 


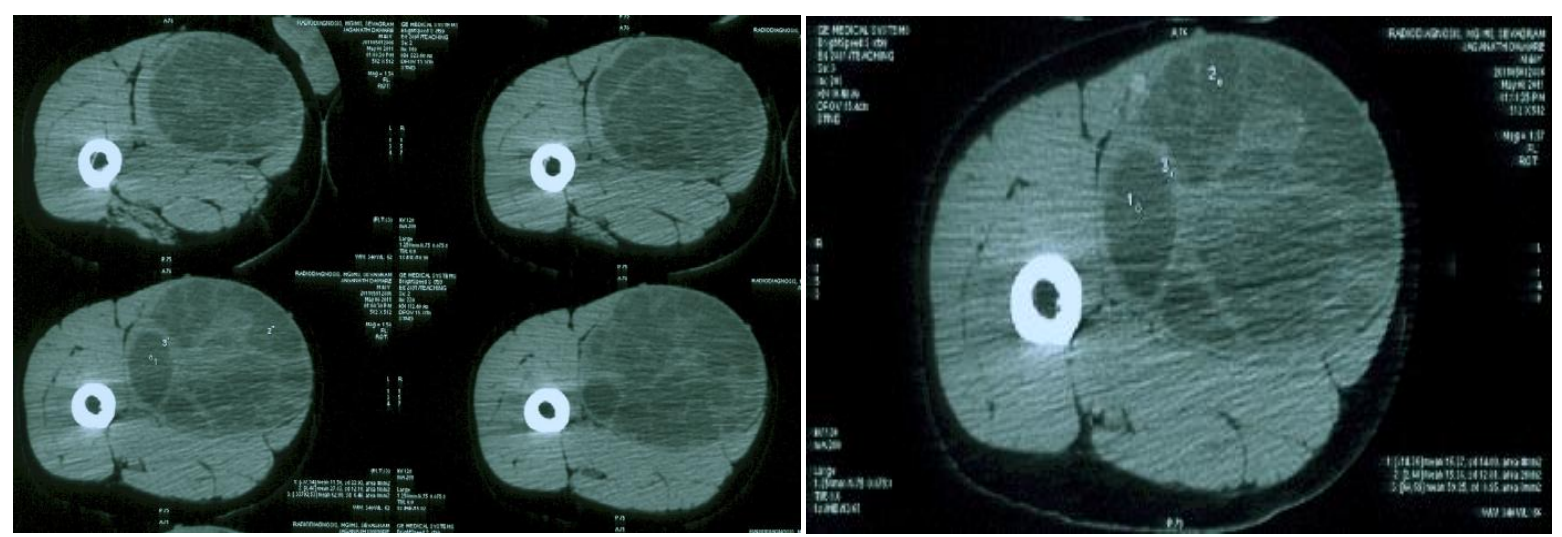

Fig. 5 a \& b: CT axial images demonstrate fluid filled density multiple cysts with nonenhancing walls in the muscular plane of right thigh

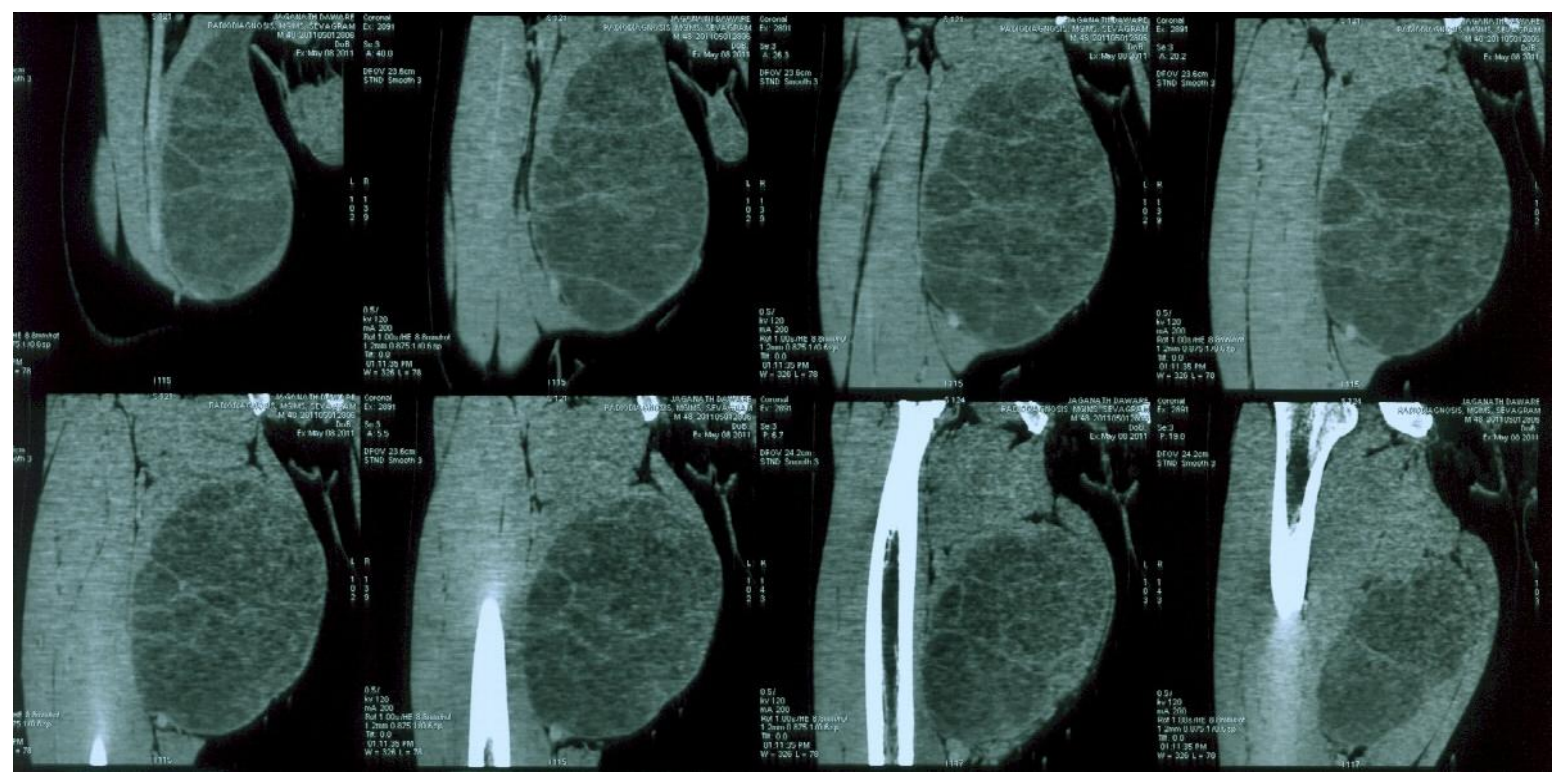

Fig. 5 c: CT Coronal Images

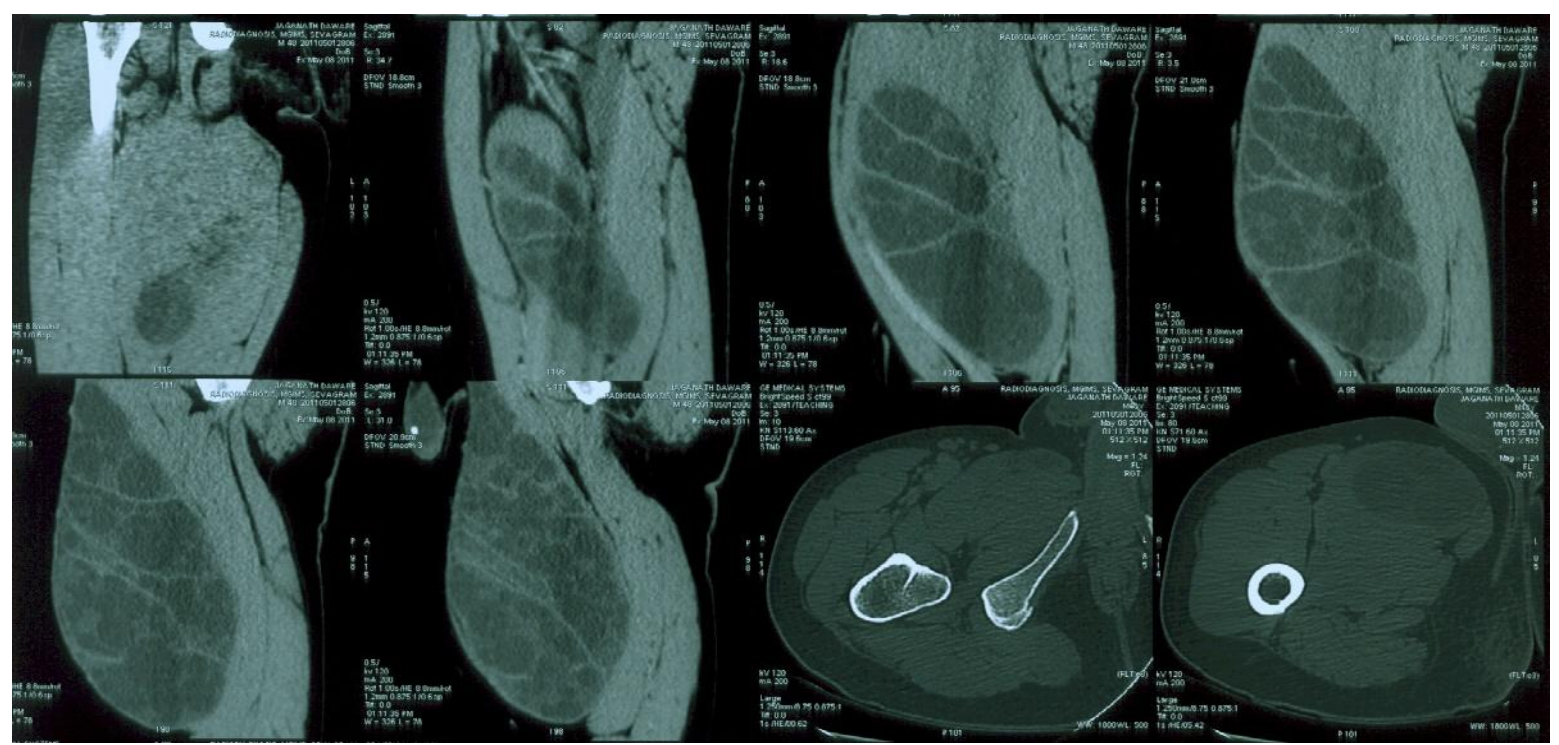

Fig. 5 d: CT Sagittal image and Bone window 
Plain Radiographs Antero-Posterior and lateral view of right thigh shows large soft tissue mass lesion on the medial aspect of right thigh. (Fig. 3)

Right thigh Ultrasonography reveals large well defined double walled cystic lesion of size $22 \times 9.6 \times 7.8 \mathrm{~cm}$ in the muscle plane of the medial aspect of right thigh, with multiple well defined anechoic cystic lesions of varying size within the cyst giving honey comb appearance. (Fig. 4 a,b )

Computed tomography of right thigh reveals multiple large round to oval fluid density cystic lesion in medial compartment of thigh involving adductor magnus muscle with multiple thin walled daughter cyst within. There is no post contrast enhancement of the lesion, no evidence of any calcification, no obvious adjacent vascular or bony involvement. (Fig. 5 a-d).

$2 \mathrm{ml}$ of clear fluid was aspirated for Fine needle aspiration cytology from swelling. Smear show acellular laminated membrane of hydatid cyst (Fig. 7).

Patient was operated and multiple pearly white cysts came out on incision. Cyst was sent for histopathological examination and Sectioning and gross examination of the cystic cavity demonstrated clear fluid with multiple daughter cysts. (Fig.6 a,b). The patient was discharged on albendazole 10 $\mathrm{mg} / \mathrm{kg}$ per day for 6 weeks.

\section{Discussion}

Primary involvement of muscles is very rare in cases of hydatid cyst; it has been reported in about $2 \%-3 \%$ of all patients. ${ }^{1}$ Isolated primary skeletal muscle involvement is rare, as the parasite has to cross pulmonary and hepatic barriers to reach the muscles. ${ }^{4,5}$
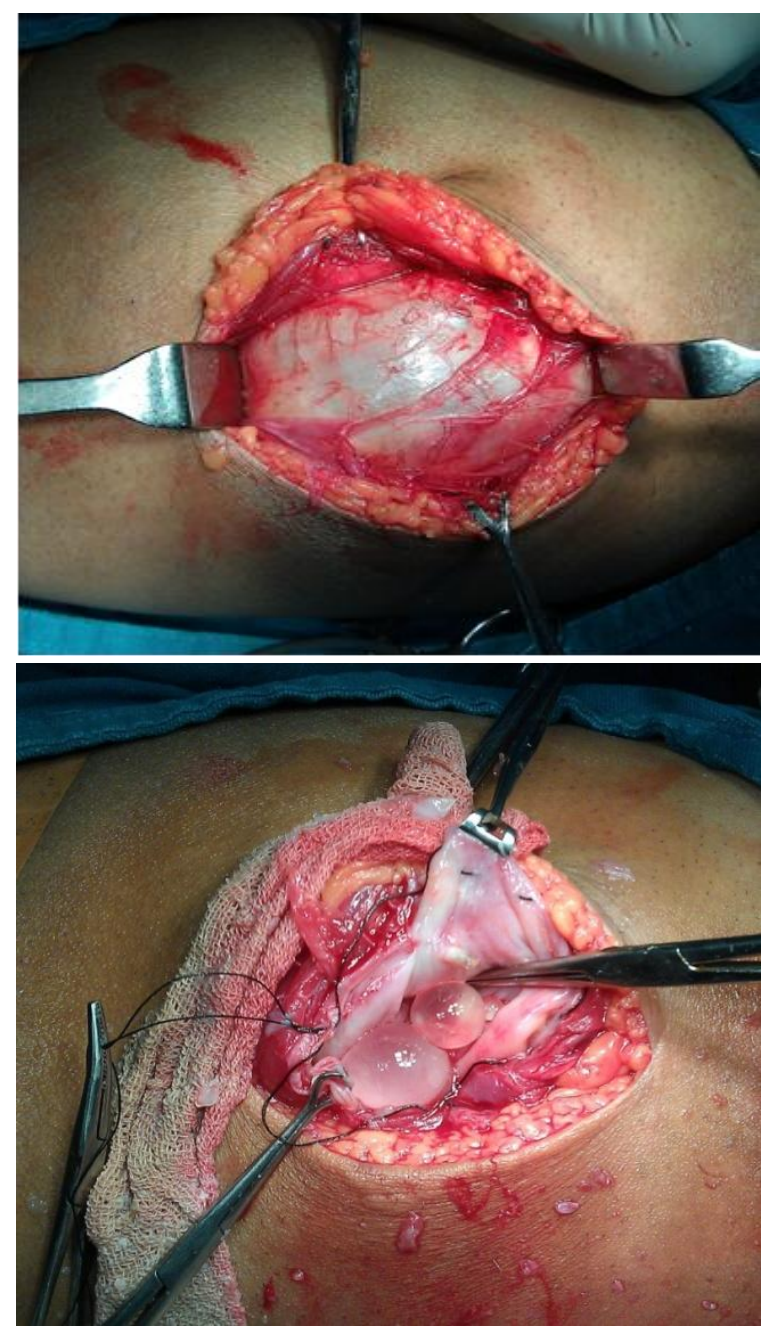

Fig. 6 a\&b: Operative photograph demonstrating the mother cyst and the multiple daughter cysts.

A hydatid cyst has three layers. The outer pericyst is composed of modified host cells that form a rigid protective zone only a few millimeters thick. The middle, laminated membrane resembles the white of a handboiled egg and is easily ruptured. It is acellular and is about $2 \mathrm{~mm}$ thick. It permits the passage of nutrients but is impervious to bacteria. Disruption of the laminated membrane predisposes to infection. The inner, germinal or germinative layer is thin and translucent. It produces the laminated 
membrane and the scolices that represent the larval stage.

Scolices are also produced by brood capsules, which are small spheres of disrupted germinal membrane. These may remain attached to the germinal membrane, but free-floating brood capsules and scolices form white sediment known as hydatid sand. Acephalocysts are sterile and unable to form scolices.

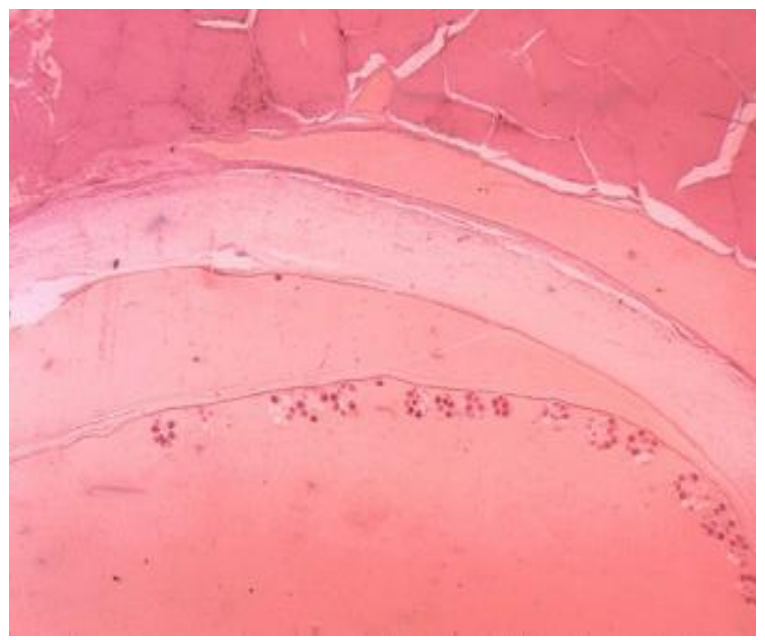

Fig. 7: Microphotograph of $\mathrm{H \&} \mathrm{E}$ stain fixed slide of specimen of Hydatid cyst,

The cyst fluid is crystal clear. It is a transudate of serum, contains proteins, and is antigenic. If released into the host's circulation, it can cause eosinophilia or anaphylaxis, although cyst rupture may be clinically silent .Serologic tests have falsepositive or false-negative rates of $15 \%-20 \%$, and positive tests may not revert to normal until several years after cyst removal. Crossreactions can occur with other parasites. Preoperative diagnosis of hydatid cysts can be made ultrasonically and confirmed by a CT scan. ${ }^{3}$ Ultrasonography should be the first diagnostic tool used for detection of hydatid disease of soft tissue. ${ }^{2}$ MRI offers no real advantage over CT scan.
Ultrasonography is useful in diagnosis, showing the size, localization, and type of the cyst. The sensitivity of ultrasonography is $95 \%$, and if vesicular fibrils are present, the sensitivity increases to $100 \%$.

Computed Tomography scan should be performed in suspicious cases or in order to determine the technique of surgery with demonstration of the relationship to adjacent organs. The CT scan has an accuracy of $98 \%$ to demonstrate the daughter cysts, and it is the best test to differentiate hydatid cysts from amebic and pyogenic cysts in the liver. ${ }^{6}$ A thin rim of calcification delineating a cyst is suggestive of an echinococcal cyst.

Several serological tests can be used for diagnosis, screening, and post-operative follow-up for recurrence. These include the hydatid immunoelectrophoresis, enzymelinked immunosorbent assay (ELISA), latex agglutination and indirect haemagglutination (IHA) test. ${ }^{7}$ The high lactic acid level in muscle tissue is considered unfavourable for parasite survival. Moreover, muscular contractions prevent fixation of larvae to the tissue. ${ }^{8}$ The sensitivity of various serological tests used for hydatid disease varies from 64 to $87 \%$. Hydatid serology is only valuable when it is positive, negative serologic test does not exclude the diagnosis. If the cyst is not complicated, serology is less sensitive but it may be useful for controlling the recurrence of cyst.

\section{Conclusion}

Hydatid disease can affect any organ in the body; the infestation may mimic a soft tissue tumor and therefore, a high suspicion of this disease is justified in any cystic neoplasm of any organ. 


\section{References}

1. Ammari FF, Khasawneh Z, Salem MK, et al. Hydatid disease of the musculoskeletal system. Surgery 1998; 124:934-7.

2. Memis A, Arkun R, Bilgen I, Ustun EE. Primary soft tissue hydatid disease: report of two cases with MRI characteristics. Eur Radiol 1999;9:11013.

3. Brown RA, Millar AIW, Steiner Z, Krige JEJ, Burkimsher D, Cywes S. Hydarid cyst of the pancreas: a case report in a child. Eur J Pediatr Surg 1995;5:121—4.

4. Garcia-Alvarez F, Torcal J, Salinas JC, et al. Musculoskeletal hydatid disease: a report of 13 cases. Acta Orthop Scand 2002; 73:227-31.
5. Engin G, Acunas B, Rozanes I, Acunas G. Hydatid disease with unusual localization. Eur Radiol 2000;10:190412.

6. Kir A, Baran E. Simultaneous operation for hydatid cyst of right lung and liver. Thorac Cardiovasc Surgeon 1995;43:624.

7. Guntz M, Coppo B, Lorimier G, Cronier P. Hydatid cyst of the liver appearing late (10-22 years) after surgical treatment of pulmonary hydatidosis. Physiopathologic problems J Chir Paris 1990;127:375-81.

8. Tatari H, Baran O, Sanlidag $\mathrm{T}$, et al. Primary intramuscular hydatidosis of supraspinatus muscle.Arch Orthop Trauma Surg 2001;121:93-4. 